Relations industrielles

Industrial Relations

\title{
Powell, Gary N., sous la direction de, Handbook of Gender and Work
}

\section{Esther Déom et Hélène Lee-Gosselin}

Volume 55, numéro 4, 2000

URI : https://id.erudit.org/iderudit/051364ar

DOI : https://doi.org/10.7202/051364ar

Aller au sommaire du numéro

Éditeur(s)

Département des relations industrielles de l'Université Laval

ISSN

0034-379X (imprimé)

1703-8138 (numérique)

Découvrir la revue

Citer ce compte rendu

Déom, E. \& Lee-Gosselin, H. (2000). Compte rendu de [Powell, Gary N., sous la direction de, Handbook of Gender and Work]. Relations industrielles / Industrial Relations, 55(4), 783-786. https://doi.org/10.7202/051364ar

Tous droits réservés @ C Département des relations industrielles de l'Universite Laval, 2000
Ce document est protégé par la loi sur le droit d'auteur. L’utilisation des services d'Érudit (y compris la reproduction) est assujettie à sa politique d'utilisation que vous pouvez consulter en ligne.

https://apropos.erudit.org/fr/usagers/politique-dutilisation/ 


\section{Handbook of Gender and Work}

sous la direction de Gary N. Powell, Thousand Oaks, Calif. : Sage Publications, 1999, 651 p., ISBN 0-7619-1355-6.

Ce livre se présente comme un volume de référence dans le domaine particulier de «femmes et travail » et il est sans contredit celui qui actuellement couvre la gamme la plus étendue des sous-thèmes ayant fait l'objet de recherches depuis les trente dernières années. Constitué de revues de littérature commandées à des chercheuses et chercheurs nord-américains et britanniques de renom sur des sujets spécifiques, cet ouvrage se veut également un outil de sensibilisation sur une diversité de questions d'intérêt liées au genre en milieu de travail. Pour atteindre ces objectifs, chaque article fait état de la situation actuelle de la théorie et de la recherche sur une variété de sujets liés au thème principal, et propose des pistes pour la recherche future.

La première partie du volume regroupe deux articles sur la signification et l'influence du « genre » et de l'origine ethnique dans le domaine du travail. On retrouve ainsi un premier chapitre qui explique notamment les différences entre le sexe (attribut biologique) et le genre (issu d'un processus de socialisation découlant du sexe). Le chapitre 2 présente d'autres caractéristiques, dont la race, le groupe ethnique et la culture, susceptibles d'influencer la conception individuelle de l'identité. On y démontre que l'influence du sexe et du genre en milieu de travail n'est pas la même pour des individus de races, de cultures et de groupes ethniques différents et que ces caractéristiques jouent différemment chez les hommes et les femmes.

La deuxième partie du volume est consacrée à l'impact des contextes économique et social sur le travail des femmes. Cinq chapitres étudient successivement l'évolution de la nature du travail durant les cinquante dernières années, le développement du secteur des services et les implications de ce changement dans la structure économique sur le travail des femmes, l'inscription des normes sociales et industrielles (masculines) dans les organisations, l'écart salarial entre hommes et femmes et enfin, la ségrégation professionnelle. Le chapitre 3, consacré au secteur des services, offre une analyse originale et très intéressante des relations de genre en se basant sur trois concepts clés reliés au sexe des offreurs de service et des clients ainsi qu'à la nature « sexuée » du service offert (vente de matériaux de construction versus soins esthétiques, par exemple). Notons également, au chapitre 5 , la contribution majeure de Maier sur la culture masculine des organisations. Ce chapitre nous éclaire sur la prégnance de la culture masculine dans les organisations et ses effets inhibiteurs sur l'accès à l'égalité des femmes.

La troisième partie présente, en huit chapitres, certains processus interpersonnels, collectifs et organisationnels qui influencent et reflètent les liens entre genre et travail. On y étudie notamment les biais sexistes dans les entrevues d'évaluation, les évaluations de rendement, l'influence sociale et le leadership, la composition sexuelle des groupes et les relations à l'intérieur des groupes de travail, le harcèlement sexuel de même que les relations «romantiques » en milieu de travail. On y voit notamment comment la discrimination en fonction du genre lors de la sélection résulte d'une dynamique complexe entre divers facteurs, soit les caractéristiques des candidats, celles de l'évaluateur et celles de la situation particulière. Il en est de même pour l'évaluation du rendement où l'effet du genre dépend d'un grand nombre de variables contextuelles. Les auteurs soulignent que la connaissance accumulée sur ces phénomènes résulte en bonne partie d'études expérimentales avec des étudiants. Davantage de 
recherches doivent donc être réalisées dans de véritables situations organisationnelles où le contexte est pris en compte pour que nous puissions mieux comprendre ces phénomènes, afin d'agir plus efficacement pour contrer la discrimination en fonction du sexe.

Un chapitre est également consacré aux leaders globaux, c'est-à-dire ces individus qui inspirent et influencent le comportement et les attitudes de personnes partout dans le monde et aux femmes qui ont exercé un leadership international à partir du plus haut poste politique de leur pays ou au sein de la haute direction des entreprises multinationales. La diversité de leurs caractéristiques et des contextes d'où elles émergent est présenté. Ce phénomène relativement récent a été sous-étudié jusqu'à maintenant et ce chapitre démontre comment on sait peu de choses sur ces femmes et leur leadership. Il suggère de nombreuses questions de recherche, tout en s'appuyant sur des commentaires provenant de plusieurs femmes « leaders globaux ».

L'avant-dernier chapitre de cette section présente un bref aperçu des différents aspects du harcèlement sexuel au travail : sa définition, les diverses perspectives selon lesquelles le phénomène a été étudié, les réponses individuelles et organisationnelles qui y sont faites et les conséquences du phénomène. Quant au dernier chapitre, il traite des liaisons « romantiques » entre personnes d'une même organisation. Après avoir discuté le faible intérêt que le monde scientifique a accordé à cette question, Powell et Foley présentent une revue de littérature et des actions prises par les organisations. Ils proposent enfin des pistes de recherches dans lesquelles ils encouragent la communauté scientifique à s'engager.

La quatrième partie traite de la problématique de la difficile conciliation entre la carrière professionnelle et la qualité de la vie. Le premier article fait le bilan des travaux sur le genre et la carrière en privilégiant la littérature sur les choix professionnels, la progression de carrière et les attitudes envers la carrière. Ce sont les études menées en particulier auprès d'hommes et de femmes gestionnaires aux États-Unis qui ont été recensées. Quelques comparaisons sont aussi réalisées avec des gestionnaires du Canada, du Japon et de l'Allemagne. Les différences entre les hommes et les femmes sont étudiées à partir des perspectives suivantes : théorie du capital humain, les rôles sociaux et les attentes, les caractéristiques de la personnalité liées au genre, à la personnalité et aux stéréotypes, et les caractéristiques organisationnelles. L'article suivant présente des réflexions sur le « plafond de verre » et tente de répondre à quatre questions : (1) pourquoi la proportion de femmes gestionnaires s'est-elle accrue au cours des dernières années ? (2) pourquoi la proportion de femmes à la haute direction des entreprises est-elle demeurée relativement faible ? (3) quelles forces permettront d'augmenter la proportion de femmes gestionnaires en général ? (4) quelles forces influenceront la proportion de femmes dans la haute direction des organisations? Un bilan des études retenant comme explications les facteurs liés au système social, à la situation et aux caractéristiques de la personne est ensuite présenté, il démontre l'utilité de chacune de ces approches pour répondre aux quatre questions et, par conséquent, la nécessité de développer des modèles explicatifs plus complexes permettant d'appréhender les interactions entre ces facteurs. Le troisième article fait le bilan de ce qui est connu sur les effets du genre dans les relations de mentorat par rapport à l'évolution de la carrière. La littérature couverte est essentiellement américaine et de nombreuses questions demeurent peu explorées, comme les effets de la diversité sur ces relations, les processus d'échange et de communication impliqués dans le mentorat, etc. Le quatrième article fait le bilan des études sur les femmes 
entrepreneures. Alors que pendant les années 1980 de nombreuses études ont abordé ce sujet alors nouveau, le rythme de production s'est ralenti considérablement pendant la décennie suivante et le chapitre a surtout le mérite d'informer les personnes qui commencent à s'intéresser à cette question. L'avant-dernier chapitre traite spécifiquement des relations entre la vie professionnelle et la vie personnelle des individus. La littérature américaine sur ce sujet a été abondante durant les dernières années, mais peu de ces recherches explorent les différences liées au genre, encore moins en contrôlant pour le type d'occupations professionnelles des hommes et des femmes. Ce chapitre propose aussi un nouveau programme de recherche, pour tirer au clair des résultats antérieurs incertains, mais aussi pour soulever de nouvelles questions. Le dernier chapitre traite du stress que vivent les femmes en emploi, en s'appuyant en bonne partie sur la littérature sur le stress occupationnel.

Enfin, la cinquième partie traite des initiatives organisationnelles en matière d'accès à l'égalité, de gestion de la diversité et de leurs impacts sur l'organisation. Les auteurs y présentent une recension exhaustive des diverses études portant sur les initiatives de gestion de l'égalité et de la diversité (que les auteurs traitent simultanément) et leurs impacts sur l'organisation. L'auteure du dernier article insiste sur la nécessité d'étudier le contexte organisationnel, soit toutes les règles et pratiques souvent informelles qui constituent des entraves ou des facteurs facilitant l'accès à l'égalité et la gestion de la diversité.

Le volume conclut par une discussion des problèmes méthodologiques liés à l'étude de la question du genre dans les organisations. Notons particulièrement la contribution de Cooper et Bosco qui présentent les résultats d'une recherche sur le traitement du thème « femmes et travail » effectuée auprès de quatre revues scientifiques américaines. On y apprend notamment que la plupart des recherches dans le domaine sont publiées dans des revues de psychologie, qu'elles sont de type quantitatif et utilisent des méthodes traditionnelles d'analyse des données. Les auteurs invitent la communauté des chercheurs à utiliser davantage les méthodes qualitatives, soucieuses de mises en contexte privilégiées par les chercheuses féministes, afin d'enrichir et de compléter les connaissances déjà accumulées.

Ce volume constitue un apport indéniable à l'étude du genre au travail. La préoccupation des auteures et auteurs de traiter souvent simultanément l'influence de la race et du sexe en milieu de travail nous rappelle qu'au Québec, les études sur le sexe ont la plupart du temps tendance à oublier le volet « race » de la discrimination au travail et l'importance d'en tenir compte pour arriver à une appréhension plus fine de la discrimination en milieu de travail. Par ailleurs, le traitement de certains thèmes, nettement moins d'actualité aux États-Unis qu'au Québec, notamment l'équité salariale, nous laisse sur notre appétit.

Il arrive souvent qu'un ouvrage de ce genre (collectif) manque d'unité dans le traitement des thèmes retenus. Le respect par les auteures et auteurs de la conception du volume ainsi que les références fréquentes à divers chapitres par les uns et les autres facilitent l'intégration des différentes parties autour du thème unificateur. L'imposante bibliographie présentée à la fin du volume constituerait d'ailleurs, en soi, une raison suffisante pour se procurer ce volume.

L'éditeur, Gary Powell, dont le premier ouvrage Women and Men in Management (1988) présentait un excellent bilan de la recherche américaine sur les femmes et la gestion, a su s'entourer de nombreux collaborateurs et collaboratrices reconnus dans le domaine. La littérature couverte par chaque auteur, loin d'être exhaustive, permet généralement une bonne couverture du 
champ, bien que ceci varie d'un chapitre à l'autre. C'était un pari difficile pour les auteurs de présenter, en une vingtaine de pages, les synthèses antérieures publiées, la recherche des vingt dernières années et de proposer des pistes pour les recherches à venir ; plusieurs l'ont réussi de façon admirable, notamment Maier sur la masculinité des organisations (Chapitre 5), Tolbert, Graham et Andrews sur les proportions et les relations hommes-femmes dans les groupes de travail (Chapitre 10), Carli et Eagly sur l'influence sociale et l'émergence du leadership (Chapitre 11) et Powell sur le plafond de verre (Chapitre 17).

Ce livre est des plus utiles et fortement recommandé tant pour les personnes qui veulent rapidement se familiariser avec le champ ou l'une des questions abordées, que pour celles qui veulent mettre en place des programmes ou des mesures adaptées aux réalités des femmes au travail et dans les organisations.

\section{Esther DÉom et Hélène Lee-GosSelin Université Laval}

\section{La confiance : approches économiques et sociologiques}

sous la direction de Christian Thuderoz, Vincent Mangematin et Denis HARrisson, Paris : Gaëtan Morin Europe, 1999, 322 p., ISBN 2-91074905-3.

La notion de confiance fait l'objet d'une redécouverte dans les sciences sociales. Elle plonge ses racines tant chez les pères de l'économie, de la sociologie que de l'anthropologie, se situant au fondement des relations économiques et du lien social. Elle a fait l'objet d'un dépoussiérage avec des penseurs comme A. Giddens, O.J. Williamson, et de façon plus générale avec ceux qui cherchent à lier économie et sociologie (par exemple : économie des conventions, théories du choix rationnel, sociologie économique...). L'ouvrage en question constitue une proposition de bilan pluridisciplinaire, ce qui en soi est une heureuse idée. De nombreuses disciplines ou quasidisciplines sont convoquées (sociologie, relations industrielles, économie, gestion), à l'aide de méthodologies relevant tant de la sociologie, de la psychologie expérimentale... que de la simple revue de littérature. L'ouvrage est le fruit de la collaboration de chercheurs français et québécois. On a malheureusement oublié de présenter les auteurs, qui défilent dans le désordre. Tous les articles sont inédits, et il ne s'agit pas d'actes hâtivement compilés. Est-ce à dire que le pari est gagné ? Pas tout à fait.
L'ouvrage est divisé en deux parties, la première à objectif théorique, la seconde à visée pratique, comprenant en tout onze contributions. Dans une introduction bien menée, Christian Thuderoz présente les grands acteurs et courants qui ont, depuis un siècle ou à peu près, construit théoriquement la notion de confiance, de même que les divers usages que l'on peut en faire dans les recherches en sciences sociales, l'objet étant de nous convaincre du caractère heuristique de la notion en temps de modernité dite avancée et de précarisation du lien social.

La première partie, à visée théorique, est constituée de cinq textes. Le premier texte, de Vincent Mangematin ( La confiance : un mode de coordination dont l'utilisation dépend de ses conditions de production ") propose de distinguer la confiance dite organisationnelle de celle dite institutionnelle, en insistant sur l'importance des conditions de production de la confiance, donc de l'ancrage sociohistorique présidant à cette production, pour conclure sur l'hybridation des différents types de confiance dès lors qu'on pénètre dans l'empirie. Williamson et Zucker sont ici principalement sollicités. Le propos 